\title{
Correction to: Efficacy and safety among second-generation and other basal insulins in adult patients with type 1 diabetes: a systematic review and network meta-analysis
}

\author{
Martin $^{1} \cdot$ Yi Zhou $^{2} \cdot$ Tatsuya Takagi $^{1} \cdot$ Yu-Shi Tian ${ }^{1}$ \\ Published online: 19 August 2021 \\ ๑) Springer-Verlag GmbH Germany, part of Springer Nature 2021
}

\section{Correction to: Naunyn-Schmiedeberg's Archives of Pharmacology \\ https://doi.org/10.1007/s00210-021-02128-9}

In the online published version of this manuscript, introduction and discussion were inadvertently published with incorrect words, i.e., level one should be level three for severe hypoglycemia and moderate to high should be low to high for nocturnal confirmed hypoglycemic events. The corrected words appear as below.

Introduction

However, their network meta-analysis on safety only concerned with severe hypoglycemia, defined as the most dangerous level (level three) by the American Diabetes Association (ADA) (International Hypoglycaemia Study Group 2017).
Discussion

In this study, we investigated level three and level two hypoglycemia (International Hypoglycaemia Study Group 2017),

Furthermore, the number of nocturnal confirmed hypoglycemic events per person-year of the second-generation basal insulins was significantly lower than NPH and first-generation basal insulins, with certainty evidence from low to high.

The corrections do not affect any other aspect of our article.

Publisher's note Springer Nature remains neutral with regard to jurisdictional claims in published maps and institutional affiliations.

The original article can be found online at https://doi.org/10.1007/ s00210-021-02128-9.

Yu-Shi Tian

yushi-tian@phs.osaka-u.ac.jp

1 Graduate School of Pharmaceutical Sciences, Osaka University, 1-6 Yamadaoka, Suita, Osaka 565-0871, Japan

2 Graduate School of Medicine, Osaka University, 2-2

Yamadaoka, Suita, Osaka 565-0871, Japan 